\title{
Framework for Safety Driving System in Connected-Vehicle Communication Networks
}

\author{
Iksoo Kim and Eun-Kyu Lee \\ Dept. of Information and Telecommunication Engineering \\ Incheon National University \\ 119 Academy-ro, Yeonsu-gu, Incheon, Korea \\ \{iskim,eklee\}@inu.ac.kr
}

\begin{abstract}
This paper proposes a conceptual framework for a safety driving system that can prevent secondary collision due to car accidents ahead on a road. The proposed framework uses sensors (shock and velocity), GPS information, video information using a Drone system and multicast transmission in connected-vehicle communication network including V2V and V2I communication network. The proposed safety driving mechanism consists of five parts, the $1^{\text {st }}$ part is a sensor part that detects shock and velocity of crashed vehicle due to collision accidents, and the $2^{\text {nd }}$ one is a GPS system that detects the position of accident. The $3^{\text {rd }}$ one is a Drone system that captures the situation video of the accidents and transmits it, and the $4^{\text {th }}$ part is a V2X communication network including multicast transmission. The last part is a safety driving server system that manages all of the information for the safety driving and controls all of devices and communication mechanism in connected-vehicle communication network.
\end{abstract}

Keywords: V2V, V2I, CVCN network, Multicast, GPS

\section{Introduction}

The roadway networks have developed gradually during the last 100 years since 1920's that the first traffic signal appeared on the roadway [1]. But in recent, the development of roadway network through combining the development of computer and wireless communication technology is remarkable. Such the convergence for IT technology and roadway network can establish more intelligent traffic network and ITS (Intelligent Transportation System). Thus, C-ITS (Cooperative ITS) can provide more rapid, comfortable and safe driving on the roadway because it supports real-time communication inter-vehicles and vehicle-to-traffic facilities.

Over the last decade, researches in vehicle communication network (VCN), Vehicular Ad-hoc Network (VANET) or V2V/V2I network have proceeded in the fields of wireless network including sensor network. One of the vehicle communication network is Car-toCar (C2C) / vehicle-to-vehicle (inter-vehicle) communication. A variety of vehicle communications support comfortable and safe driving on the roadway. Connected vehicles network is more intelligent and safety form of C2C [2, 3, 4].

The connected vehicles communication network (CVCN) may classify into some main fields that communicate with Vehicle-to-Vehicle (V2V) in infra-structureless network/or Mobile Ad-hoc Network (MANET), Vehicle-to-Infrastructure (V2I)/or Vehicle-toRoadside units (V2R) with the aid of communication-infra such as the roadside units (RSU) and 3G/4G cellular-network (cellular phone), and Vehicle-to-Nomadic (portable) devices (V2N) such as notebook computers, equipped on-boarded a variety of sensors, tablet PCs, navigators and MP3 players via wireless communications network. And the connected vehicles network has developed as an important area of ITS in the type of 
wireless using smart devices including a variety of sensors equipped on-board in vehicles [3].

The communication technologies of connected vehicles network are $3 \mathrm{G} / 4 \mathrm{G}$ cellular (cellular phone), Wi-Fi and $5.9 \mathrm{GHz}$ DSRC (dedicated short range communication) which allocates $75 \mathrm{MHz}$ bandwidth and works with approximate range of $1000 \mathrm{~m}$. WAVE (Wireless Access in Vehicular Environments) is a set of standards to support multichannel guaranteed $1 \mathrm{Mbps}$ at the speed of $200 \mathrm{Km} / \mathrm{sec}$ and to allow cooperative and robust safety critical applications in V2V and V2I wireless communication, and the WAVE is the one of the best promising communication technology for connected vehicle network [5-6].

This paper proposes multicast transmission technique in vehicle communication network for stringent video information such as the scene of the vehicle accident ahead in vehicular communication network and providing video streams for entertainment $[7,8,9]$. The rest of this paper is as follows: Section 2 presents overview of connected vehicle application and technology, and Section 3 deals with the structure of safety driving system in connected-vehicle communication networks. Section 4 discusses operation mechanism for the framework of safety driving system in connected-vehicle communication networks. Finally, we discuss our conclusion.

\section{Overview: Connected Vehicle Application and Communication Net- Work}

\subsection{Connected Vehicle Application}

The fields of connected vehicle applications consist of safety applications, mobility applications and environmental applications, but these application fields are not limited only to drivers that equipped on-board (including a variety of sensors) in vehicles with smart technologies (including communication technologies: WiFi, DSRC, 3G/4G celluar and devices: handphone, notebook computers, tablet PCs and portable navigators).

The field of safety applications reduces/eliminates/avoids vehicle crashes including prevention secondary collision accident due to heavy vehicle crashes accidents or construction zone or weather conditions (fog, snow or icy road) ahead on the roadway and highway. The World Health Organization (WHO) announces that more than 1.24 million people were died by car accidents in a year, that is 3,400 people died a day and additional 20-50 million people are injured or disabled that reported WHO's Global Status Report on Road Safety 2013. The victims' rate of working aged people (age $17 \sim 44$ ) among the deaths by car accidents is more than 50\%. And almost 33,000 people were died due to car accidents and 2.3 million people were injured in the United States in 2013. 1,600 kids under 15 years and almost 8,000 fatalities ages 16-20 are died in car accidents and the amount of damage for car accidents on the roadway is reached more than $\$ 230$ billion per each year in the United States [10-11].

In Figure 1, the blue bar graph shows fatalities and red band graph shows fatality rate per $100 \mathrm{M}$ vehicle miles traveled by year 2000 to 2013 that published annual report of National Center for Statistics and Analysis [11]. The figure also demonstrates that even though fatalities and fatality rate due to in car accidents have decreased very slowly since year 2007, those facts in terms of social loss is very serious problem. Thus, the most important thing in safety driving applications is to reduce/eliminate/avoid of vehicle accidents. 


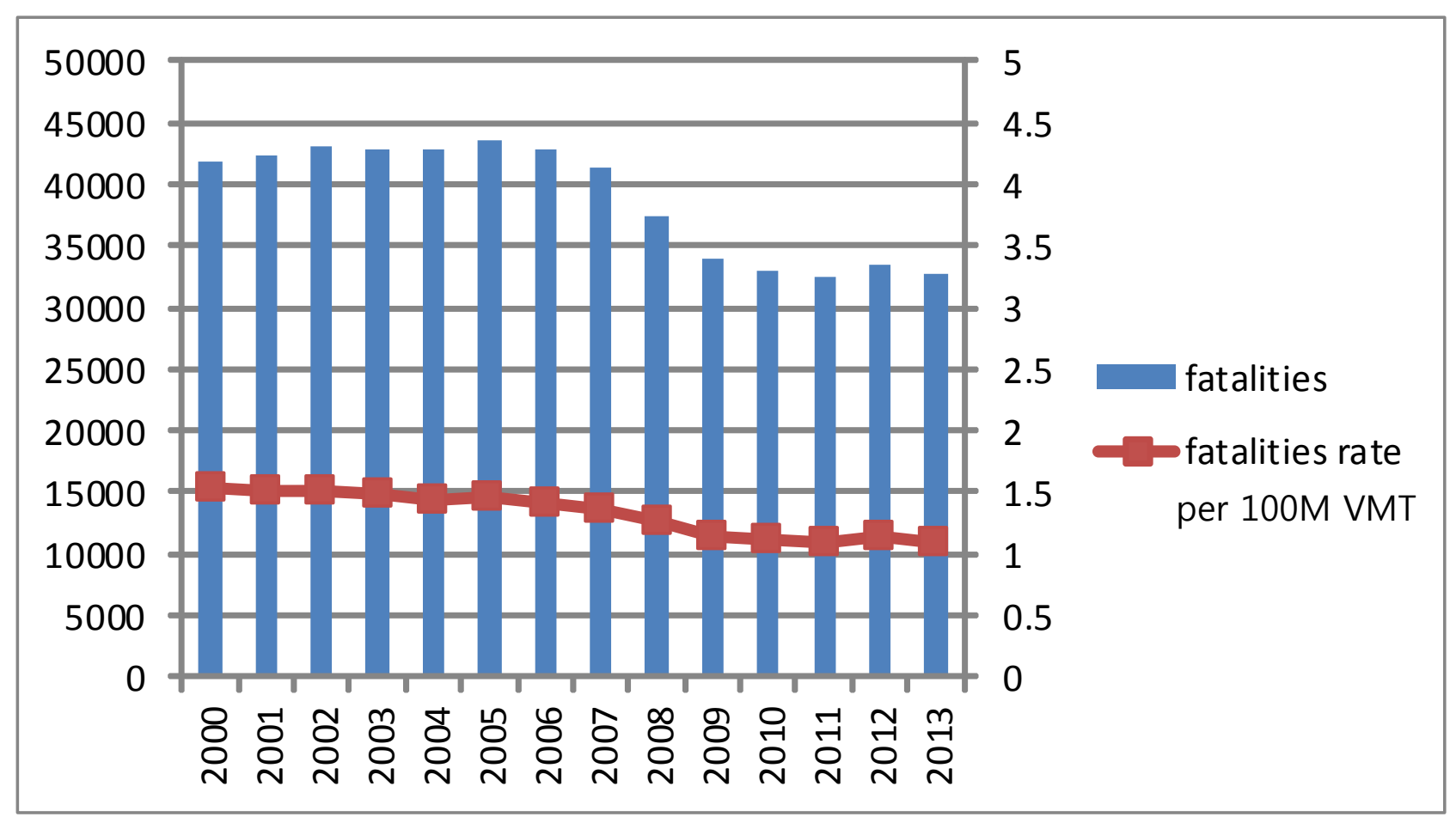

\section{Figure 1. Fatalities and Fatality Rate per 100M Vehicle Miles Traveled by Year (Source: FARS 2000-2013, 2013 Annual Report File of NHTSA's Na- tional Center for Statistics and Analysis [11])}

There are two types of safety concept in connected vehicle applications, those are soft safety application and hard one. The soft safety application is the area of increasing safety driving such as warning about road conditions including icy roads, construction zones, and traffic information(traffic jams) on forwarding direction on the road and highway. Thus, the hazards in the soft safety applications are not imminent. But the hazards in the hard safety applications are stringent. And the hard safety application is the area of avoiding vehicle crash accidents and forward collision warning to avoid secondary collision, and minimizing the damage when these vehicle accidents become unavoidable. The field of mobility applications is designed to capture real-time data such as vehicles accidents, variable speed limit signs, construction signs, lane-drop signs, congestion information and detour route information including re-routing information transmitted from the traffic (transportation management) servers that managed and controlled by traffic management office and the number of vehicles on the roadway (highway). And the field of environmental applications is to support green transportation such as fuel-efficient and ecofriendly driving to reduce the amount of $\mathrm{CO}_{2}$ emission during vehicle trip. Especially, the fuel-efficient driving can reduce fuel consumption and prevent environmental pollution $[4,12]$.

\subsection{Network-bandwidth for Connected Vehicle Communication Network}

In wireless networks including vehicle-based networks, one of the common critical problems is insufficient network resources. The shortage of network bandwidth is a main critical problem in the case of transmission of multimedia data including video, audio, MP3 and web-games. Thus, the transmission mechanism of the situation videos of a vehicle-accidents or road conditions using a Drone system of the proposed conceptual frame- 
work in this paper is affected since the insufficient network resources in the connected vehicles communication network $(\mathrm{CVCN})$. Also, the cutoff of service/or connection and the load of transportation or infotainment server are another problems in CVCN and wireless networks $[3,7,9]$.

In order to solve the insufficient network bandwidth problem, this paper exploits multicast transmission that help us use network bandwidth effectively in CVCN and reduce the load of transportation server. We focus on safety applications in connected vehicle network - more specially, avoidance of secondary collision accident due to heavy vehicle crashes ahead on the roads and highway. In the avoidance process, vehicles on the roadway are informed of vivid scenes of an accident or road conditions in real-time using multicast transmission technique, which is expected to reduce predicted accidents. In this way, the proposed mechanism can provide safer and more comfortable driving without consuming deficient network resources because it sends more vivid multimedia data using multicast transmission.

\section{The Structure of Safety Driving System in Connected-Vehicle Communication Networks}

Figure 2 shows the schematic diagram of connectivity in CVCN. The connectivity is composed of some components: the connectivity for the communication among the all kinds of vehicles whether communication infra exist or not, for supporting the safety and comfortable driving to drivers, for a variety wireless devices and sensors, and for transportation/infotainment server in CVCN network [5].

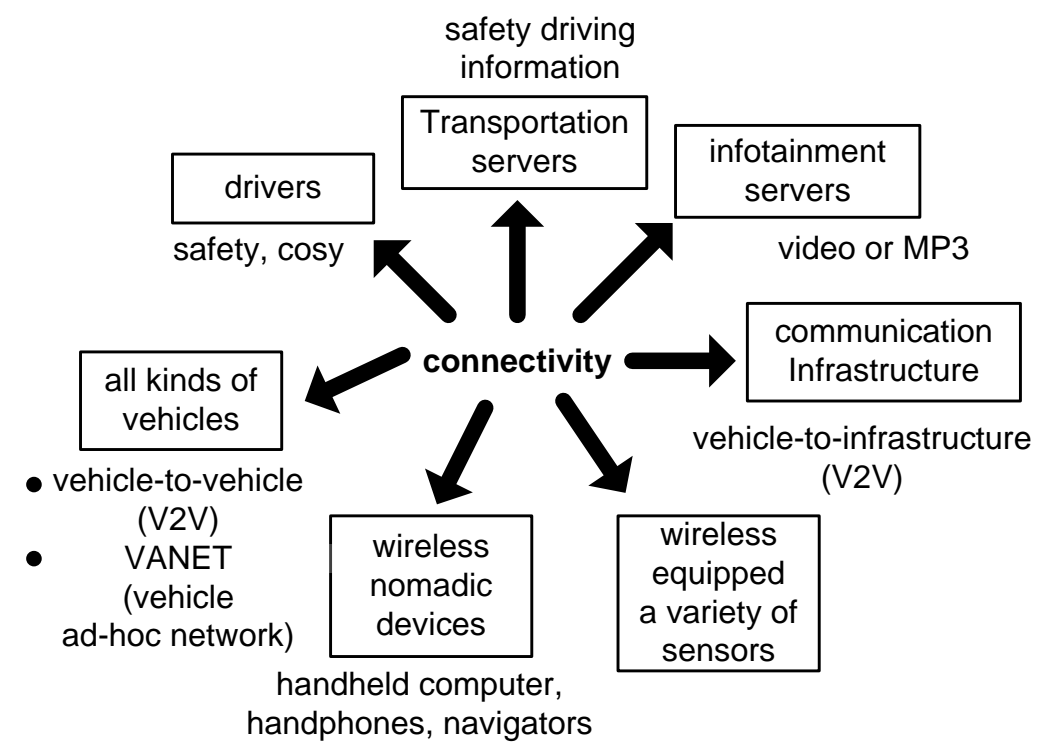

Figure 2. The Schematic Diagram of Connectivity in CVCN

Figure 3 illustrates the structure of the proposed framework of the safety driving system in CVCN using DRONE system and multicast mechanism for the video scene of crashed vehicles' position on the roadway. We note that we extend our previous structure in [7] for this work. The proposed safety application system in CVCN with DRONEs, as shown in the same figure, consists of transportation and infotainment server, some localvehicle-networks (LVNs), DRONEs with camera, many driving vehicles in CVCN network and a satellite connected through the Internet. The CVCN network may encompass all kinds of vehicle networks including V2V, V2I, V2N and VANET. 




Connected Vehicle Network with DRONEs

Figure 3. The Structure of the Proposed Safety Application System in (CVCN) with DRONEs

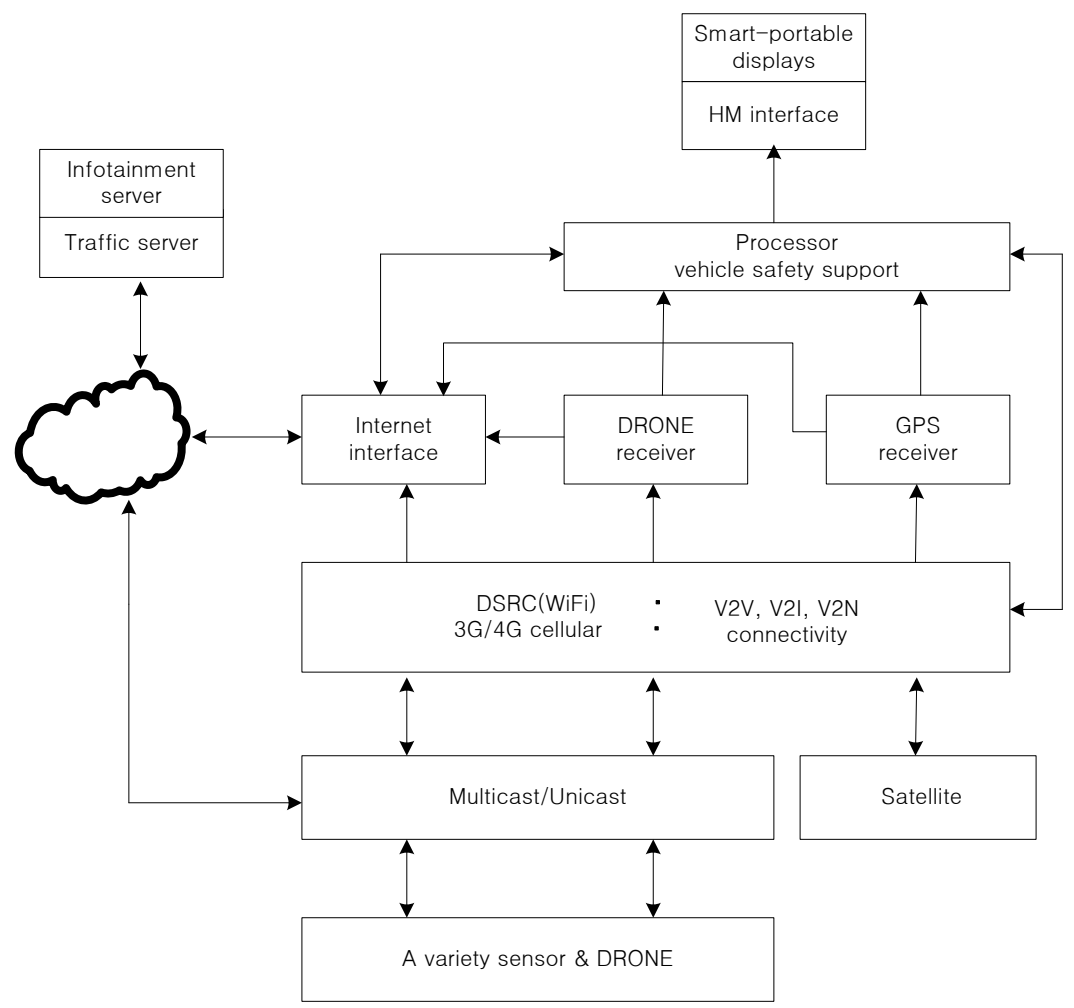

Figure 4. The Building Block of Safety Application System in CVCN 


\section{Operation of the Framework of the Safety Driving System in Con- nected Vehicle Communication Network}

\subsection{The Operation Mechanism for Driving Vehicles}

The proposed operation mechanism for driving vehicles is described in detail as follows.

i) The SRSU (smart road side unit) assigns a specific IP address to the driving vehicle when it enters the connected-vehicle communication network on the roadway.

ii) The driving vehicle accesses the transportation server through SRSU connected.

iii) The driving vehicle receives two multicast group IPs (mcast_add_i and mcast_add_j) for the safety and comfortable driving from the transportation server.

$i v)$ The driving vehicle joins the two received multicast group IPs in order to receive real-time information transmitted from the transportation server and to send captured information to other (rear) vehicles on the roadway and transportation server.

v) The driving vehicle sends information to the rear vehicles and the transportation server when it detects more than marginal value of weather conditions or road conditions (icy road or construction zone) or other traffic conditions from a number sensors equipped on-board with the vehicle.

vi-i) The driving vehicle receives the weather condition such as fog, rain, snowing or other traffic conditions information in addition to driving position data from forwarding driving vehicles.

vi-ii) The shock sensor in the vehicle detects shock when the driving vehicle collides. At the same time, the velocity/speed sensor in the vehicle checks whether the vehicle stop or not.

vii) The safety driving system equipped on-board with the vehicle transmits the position information of collision with the aids of GPS system to the transportation server and rear driving vehicles if the vehicle detects more than marginal value of shock, vehicle stop and captured real-time weather condition information.

viii) The safety driving system in the vehicle and the transportation server notify its collision position to the traffic police station and the agent of vehicle insurance.

ix) The transportation server commands that launch to captured the scene of collision or critical road-conditioned areas to a specific the DRONE system.

x) The transportation server sends the captured all of the information from the driving vehicle to the vehicles on the road using the generated multicast group IP (mcast_add_j ) already.

(All of the driving vehicles can be serviced by joining with the multicast group IP (mcast_add_j ))

xi) All of the driving vehicles computes the distance between current position and the crashed vehicle position or critical road-conditioned areas captured with the multicast group IP (mcast_add_j ) using GPS information.

xii) All of the driving vehicles on the roadway decelerate to safe driving according to the distance computed in step (xi).

In step iii) of the operation mechanism for driving vehicles as described above, the transmitted two multicast group IPs for the safety driving from the transportation server are $m c a s t \_a d d \_i$ and $m c a s t \_a d d \_j$. The $m c a s t \_a d d \_i$ is a multicast group address to use when the driving vehicles have information(the scene of collision or critical roadconditioned areas) for safety driving, and the mcast_add_j is another one to use when the 
transportation sever and infotainment server have information for safety driving and comfortable driving, respectively. Thus, the driving vehicles on the roadway can be supported the safety driving service from the transportation sever by joining multicast group addresses.

\subsection{The Operation Mechanism for DRONE System}

The proposed operation mechanism for a DRONE systems is described in detail as follows.

i) The DRONE system receives the crashed position or critical road-conditioned areas information (GPS information) from transportation server.

ii) The DRONE system launches itself to the crashed position or critical road-conditioned areas with the aids of GPS system equipped on-board.

iii) The DRONE system actuates its camera to capture the scene of crashed position as soon as it has arrived the crashed position or critical road-conditioned areas.

iv) The DRONE system transmits the captured scene of crashed position to the transportation server through the CVCN.

v) The DRONE system returns to the launching site when it finishes capture and transfer operation.

The step (i) is not limited to the crashed or collision position but the positions are various locations such as icy roadway, fog area, construction area and jamming locations. Thus vehicle drivers on the roadway can recognize traffic and road condition including lane-drop due to accidents or construction in advance because the DRONE system captures the various critical scenes of a specific location.

The DRONE system consists of a number of DRONEs that is located at interval of about $2 \mathrm{Km}$. Launching a specific DRONE depends on the distance between the launching sites and concerned location. Thus, the decision of the launching of a specific DRONE is done by transportation server, generally. Of course that may be done by DRONEs on the roadway because of use GPS information and multicast transmission.

\subsection{The Operation Mechanism for Transportation Server in Connected Vehicle Network}

The proposed operation mechanism for a transportation server in the connected vehicle network is described in detail as follows.

i) The transportation server assigns a specific IP address to the driving vehicles when they request accessing through SRSU

ii) The transportation server sends two multicast group IPs (mcast_add_i and mcast_add_j) for the safety and comfortable driving as mentioned step (iii) in the operation mechanism for driving vehicles

iii) The transportation server sends general traffic information in order to safety driving to all the driving vehicles with multicast group IP (mcast_add_i)

iii-i) The transportation server sends real-time information updated to all of the driving vehicles on the roadway with multicast group IP.

iii-ii) The transportation server computes the position of the crashed vehicle using GPS positioning information received from the crashed vehicle.

iv) The transportation server transmits the position of the crashed vehicle computed in step (iii-ii) to all of the vehicles on the roadway. 
v) The transportation server selects DRONE located the nearest to the crashed vehicle and issues command to launch the DRONE.

vi) The transportation server sends the position of the crashed vehicle to traffic office, the nearest hospital and tow truck (wrecker).

vii) The transportation server issues command to capture the scene of crash to DRONE arrived the position of the crashed vehicle.

viii) The transportation server receives the scenes of crash from the DRONE and transmits them to all of the vehicles on the roadway with multicast group IP.

ix) The transportation server issues command to the DRONE to return its launching site.

$x)$ The transportation server does not transmit the scenes of crashed position to all of the vehicles on the roadway with multicast group IP.

\section{Conclusion}

This paper proposes the framework of the safety driving system in connected-vehicle communication network using DRONE system. This conceptual system for safe driving protects secondary car accidents ahead on the road using shock and velocity sensors on crashed vehicle. This proposed mechanism can provide more safe driving information in real-time because it sends more vivid scenes (accident spots, foggy and icing areas) for safety driving with the aid of Drone to the driving cars on the road. This proposed system can use more effectively connected vehicle communication network (CVCN) bandwidth since multimedia data (more vivid scenes) are transmitted using multicast transmission. The research for implementation of this proposed safety system in CVCN is in progress.

\section{Acknowledgements}

This work was supported by the Incheon National University Research Grant in 2015.

\section{References}

[1] World Road Association and FISITA, "The Connected Vehicle", at http://www.fisita.com/publications/connectedvehicle.pdf

[2] M. Jerbi, S. Senouci, Y. Ghamri-Doudane and M. Cherif, "Vehicular Communications Networks: Current Trends and Challenges," Handbook of Research on Next Generation Networks and Ubiquitous Computing, IGI-Global, (2009).

[3] G. Karagiannis, O. Altintas, E. Ekici, G. J. Heijenk, B. Jarupan, K. Lin and T. Weil, "Vehicular networking: A survey and tutorial on requirements, architectures, challenges, standards and solutions", IEEE Communications Surveys \& Tutorials, ISSN 1553-877X, vol. 13, no. 4, (2011),pp. 584-616.

[4] NHTSA, "USDOT Connected Vehicle Research Program: Vehicle-to-vehicle Safety Application Research Plan", DOT HS 811373 October (2011).

[5] Lu N., Cheng N., Zhang N., Shen X. and Mark J., "Connected vehicles: solutions and challenges", IEEE Internet Things Journal, vol. 1, no. 4, (2014), pp. 289-299.

[6] S. A. M. Ahmed, S. H.S. Ariffin and N. Fisal, "Overview of wireless access in vehicular environment (wave) protocols and standards", Indian Journal of Science and Technology, vol. 6, no. 7, (2013), pp. 4994-5001.

[7] Y. Woo and I. Kim, "Vehicle-Based Multicast Communication System for Live-Video", International Journal of Multimedia and Ubiquitous Engineering, vol. 8, no.5, (2013), pp.325-332.

[8] U. Dwivedi, A. Rajawati and A. Upadhyay, "Characterize an approach to improve performance of multimedia services over vehicular ad-hoc network", J. of Information System and Communication. ISSN: 0976-8742 \& E-ISSN: 0976-8750, vol. 3, (2012).

[9] B. Kim, Y. Woo and I. Kim, "Multimedia Service using CSMA/GPS in Vehicular Communication Network", International Journal of Multimedia and Ubiquitous Engineering, vol. 9, no. 11, (2014), pp. 123-132.

[10] "WHO's Global Status Report on Road Safety", (2013).

[11] "NHTSA's Fatality Analysis Reporting System (FARS) Encyclopedia", at http://wwwfars.nhtsa.dot.gov/Main/index.aspx 
[12] "U.S. Department of Transportation (US DOT), ITS Research Fact Sheets - Transit Connected Vehicle Research Program", at http://www.its.dot.gov/factsheets/transit_connectedvehicle.htm.

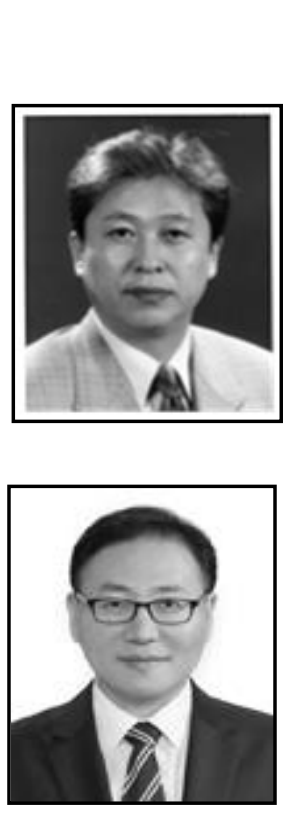

\section{Author}

Iksoo Kim, he received the B.S., M.S., and Ph.D. degrees in Electronics Engineering from Dongguk University, Korea, in 1978, 1981, and 1985, respectively. Since 1988, he joined in Incheon National University as a professor. He was a visiting professor on North Carolina State University and California State University, Sacramento in 1993 and 2004, respectively. His major interests are web-caching, multicast, multimedia system, MANET and VANET.

Eun-Kyu Lee, he is an assistant professor in the Department of Information and Telecomm. Engineering of Incheon National University, Korea. He has been involved in many research projects in the fields of smart grid, vehicular network, location-based services, and Telematics. He received Ph.D. in Computer Science from UCLA in 2014. His research interest includes the Internet of Things, cyberphysical systems, wireless networks, cybersecurity, middleware systems, and internet computing. 
International Journal of Multimedia and Ubiquitous Engineering Vol.10, No.10 (2015) 